\title{
REPRESENTAÇÃO CIENTÍFICA A PARTIR DAS MEMÓRIAS DE INVESTIGAÇÃO: EXPERIÊNCIA NA UNIVERSIDADE ESTADUAL DE CAMPINAS
}

Adilson Luiz Pinto

Beatriz-Ainhize Rodriguez Barquín

\section{Resumo}

Esta pesquisa trata de analisar a produção científica da Unicamp, tendo como base a literatura branca e cinzenta produzida pelo seu corpo docente e objetiva verificar o vínculo da produção com o seu financiamento. Em relação ao método adotado, foram levantadas informações dos anuários de pesquisa (ou memórias) e, posteriormente, lançadas em bases de dados, por produtividade e por grupos de pesquisa, construídas para tal fim. Este procedimento visou à aplicação de um filtro às informações, de modo a gerar os indicadores em input e output, segundo a análise bibliométrica. Os resultados alcançados, revelaram crescimento científico no período estudado (1995/2002), principalmente nos anos de 1998 e 2002, onde todas as áreas apresentaram evolução evidente, isto é, conseguiram desenvolver números positivos para a consolidação científica da instituição e contribuir para os indicadores do Institute for Scientific Information.

\section{Palavras-chave}

Produção científica - Unicamp; Bibliometria; Tipologia documental; Literatura branca e cinzenta.

\section{SCIENTIFIC REPRESENTATION FROM THE MEMORIES INVESTIGATION: AN EXPERIENCE IN STATE UIVERSITY OF CAMPINAS}

\begin{abstract}
This research analyzes the scientific production of the Unicamp, starting from the white and grey literature produced by the teachers from this institution, to observe the link of the scientific production with the external financing. In relation to the adopted method, all the existing information was rescued from yearbooks of research, which was put in a database (data about productivity and research groups), to filter the information and generate productivity science indicators in outputs and inputs, according to bibliometric analysis. The results of the research demonstrated that there was a relative scientific growth on the established period (1995/2002), mainly in 1998 and 2002, when the evolution of all the areas was evident, reaching significant numbers for the institutional scientific productivity and collaborating with the indicators of Institute for Scientific Information.
\end{abstract}

\section{Key words}

Scientific production - Unicamp; Bibliometrics; Documental typology; Literature, white and grey. 


\section{INTRODUÇÃO}

O estudo da produção científica implica o desenvolvimento de uma atividade específica, seja através de técnicas sofisticadas da Matemática, seja simplesmente por meio de uma análise de dados. Seus resultados podem representar científicamente um país, uma instituição, um determinado número de investigadores e uma base de conhecimento ${ }^{1}$.

Ao analisar um continente, um país ou até mesmo uma instituição que não tenha, por questão de temática e até mesmo por amplitude de sua investigação, sua produção indexada em bases de conhecimento internacional ${ }^{2}$, deve-se recorrer a outros tipos de fonte, como os currícula e as memórias científicas de seus pesquisadores.

Para este estudo, foram averiguados os dados das referidas memórias da Universidade Estadual de Campinas (Unicamp), visando a analisar quantos grupos de pesquisa estão consolidados na instituição e quantos professores estão envolvidos na produtividade científica (input); por outro lado, buscou-se analisar a produção de artigos, livros e trabalhos publicados em eventos (output), através do Sistema de Informação de Pesquisa e Extensão (UNICAMP, 2003).

Este trabalho se baseia no pressuposto de que a universidade é o principal elo entre os indicadores de input e output, sendo responsável por esta atividade, em nível nacional (OHIRA, 1997). Por outro lado, a escolha da Unicamp deve-se ao fato de ser uma das instituições de pesquisa com maior índice de produção no país (PEREIRA, 2005).

Como ponto central deste trabalho, será demonstrado que as memórias de pesquisa podem constituir um recurso relevante na análise da ciência, (i) por fornecerem informação sobre publicação de livros, teses e trabalhos apresentados em eventos, (ii) informações sobre artigos científicos por temática e (iii) por poderem informar sobre recursos financeiros e sobre a mobilidade de uma determinada instituição.

\footnotetext{
${ }^{1}$ Exemplos: ISI (Web of Knowledge - Thompson), Pascal (INIST - Françac) e outras iniciativas como Latindex (México), Pro-Quest (EUA), entre outras.

${ }^{2}$ Por áreas de conhecimento: Medicina (Medline), Biol.ogia (BIOSYS), Educação (ERIC), Economia (Econlith). Nestes casos, é aconselhável que se publique dentro das bases de dados que contemplem temáticas epecíficas, do mesmo modo que os estudos de âmbito local sejam publicados em revistas nacionais também específícas.
} 
Em relação à metodologia, o percurso eleito foi a coleta de informações sobre a produção científica dos investigadores registrados nos anuários de pesquisa ${ }^{3}$, entre 1995 e 2002. Os dados sobre a referida produção foram posteriormente organizados em um banco de dados, especialmente construído para este fim, e quantificados automaticamente.

A extração exaustiva dos dados constitui um fator positivo do presente estudo, na medida que apresentou resultados significativos. No entanto, cumpre registrar que temse como fator determinante para o êxito do levantamento a qualidade da Universidade eleita, a qual revela uma produtividade sólida, constante e com uma particularidade brasileira, isto é, voltada a todos os tipos de pesquisa, seja através de artigos indexados no SSCI/SCI ${ }^{4}$, de livro ou de trabalhos publicados em eventos (literatura branca e cinzenta).

\section{ANTECEDENTES HISTÓRICOS E TÉCNICAS PARA A GERAÇÃo DE INDICADORES CIENTÍFICOS}

Nos países periféricos, a produção científica se concretiza no meio acadêmico pelas mãos dos docentes, principalmente os de carreira e aqueles vinculados aos Programas de Pós-graduação (SCHWARTZMAN, 1993).

Esta ação produtiva que as universidades desempenham através de seus docentes fez com que a produção brasileira indexada no ISI se elevasse de forma extraordinária e por tal razão os organismos oficiais do país começaram a preocupar-se por seu desempenho dentro dessa base de conhecimento (LOUZADA, 2002).

No período de 1981 a 2000, duplicou o volume de artigos oriundos de todos os países. No caso do Brasil, a produção também cresceu, passando de 1.889 trabalhos indexados no início do período, para 9.511 em 2000, o que representa um crescimento de 403,49\%. Este dado coloca o Brasil entre os 17 países mais importantes cientificamente

3 Material gerado e distribuído pela Pró-Reitoria de Pesquisa e Pós-graduação da Unicamp e disponível na página do Sistema de Informação de Pesquisa e Extensão (Sipex).

4 Bases de conhecimento do Institute for Science Information (ISI), denominadas Science Citation Index (para ciências em geral) e Social Science Citation Index (para a área de Humanidades e Ciências Sociais). 
naquela base e com um destaque substancial em crescimento científico, o que lhe confere a nona posição no ranking geral do ISI. Entretanto, a produção brasileira indexada no período de 2000 e 2001, dentro de ISI, cresceu apenas 11\%, passando de 9.511 para 10.555 artigos $^{5}$. De acordo com a informação da produção mundial indexada no SCI/SSCI, no mesmo período (1981-2002), o crescimento foi da ordem de 2,8\%, passando de 714.171 a 734.248 artigos (NATURE, 2002), decompostos pelos principais países produtores.

Estas informações ressaltam a ascensão da produção científica brasileira diante dos principais produtores de ciência no mundo (visibilidade científica), tendo um enfoque muito interessante a partir de 1995 (LOUZADA, 2002), quando a atividade passa a ser mais intensa. Com 10.555 artigos no período, o Brasil se defronta com a França (40.645), Itália (24.665), Inglaterra (52.730), Coréia do Sul (5.403), Japão (58.640), Alemanha (53.159) e China (13.641), que apresentam um maior amadurecimento nesse aspecto segundo informações do ISI.net.

A visibilidade científica, segundo as bases de conhecimento, é um fator significativo para demonstrar o grau de evolução dos países. No entanto, cumpre acrescentar que estas fontes contemplam, em sua maioria, revistas em língua inglesa e poucas em língua portuguesa. Por este motivo, muitos investigadores brasileiros dão preferência a publicar suas pesquisas em revistas nacionais, tendo em vista: (i) que suas investigações estão centradas no ambiente nacional e (ii) que seu público-alvo são os pensadores brasileiros.

Isto não significa dizer que o investigador brasileiro não possa divulgar seus trabalhos em ambiente internacional, principalmente se estes tratarem de temas das chamadas ciências-chave (como Química, Física e Medicina), por sua aplicação adequar-se a qualquer ambiente.

Em relação ao período estudado (1995 a 2002), deve ser ressaltado que o País começa a ter um destaque mais significativo nas bases internacionais, principalmente nos pacotes ISI. Por outro lado, é importante mencionar que, paralelamente ao labor das bases

\footnotetext{
${ }^{5}$ Quadro estatístico do CNPq sobre a produção científica mundial dentro do Institute for Scientific Information (www.cnpq.br).
} 
internacionais, órgãos do Governo Federal e as fundações estaduais de pesquisa começam a divulgar os índices científicos brasileiros, destacando o período indicado como o mais frutífero da história da ciência nacional.

Fortalecendo a inicitiva dos órgãos científicos (governo federal e fundações estaduais de pesquisa), neste mesmo período o país implanta algumas iniciativas enriquecedoras de divulgação da produção nacional para a América Latina, Caribe e parte da Europa de língua latina, implementando o sistema SciELO ${ }^{6}$ e o Prossiga ${ }^{7}$.

Em paralelo e motivados por este novo cenário, os pesquisadores brasileiros buscam recursos para estudar e representar a ciência nacional em números, utilizando técnicas métricas.

As técnicas bibliométricas mais utilizadas na atualidade pelos cientistas brasileiros e que serviram de base para o presente estudo, são as de:

- Lotka (1926), que trabalhava com os indicadores de input (número de docentes e recursos financeiros) e output (número de publicações), representando os autores com maior contribuição para uma determinada área científica;

- Garfield (1955), que aproveitou todas as teorias anteriores para desenvolver um recurso de normalização do conteúdo bibliográfico;

- Price (1963), que trabalhou com o estudo do crescimento exponencial das ciências;

- Schwartzman (1993), que descreve os caminhos do desenvolvimento nacional através da produção científica e ressalta a importância de publicar em revistas que estão indexadas em bases de conhecimento;

- Población, Noronha e Currás (1995), que descrevem a importância de analisar a produção científica sob dois aspectos: literatura branca e literatura cinzenta.

\section{MÉTODO}

${ }^{6}$ Scientific Electronic Library Online (SciELO) é uma biblioteca eletrônica que abarca coleção selecionada de revistas científicas brasileiras também se constitui em uma metodologia de avaliação de revista científicas (www.sceilo.br).

${ }^{7}$ Prossiga é um projeto do Ministério da Ciência e Tecnologia e tem por objetivo promover a criação e o uso de serviços de informação especializada na Internet, voltados para as áreas prioritárias, assim como estimular o uso de veículos eletrônicos de comunicação pelas comunidades dessas áreas. 
Para fins deste estudo, foram levantados os dados dos grupos de pesquisa da Unicamp, consolidados no período eleito (1995/2002). O referido período contou, segundo as memórias, com uma média de 1.900 docentes e pesquisadores/ano, nas áreas de Ciências Agrárias, Ciências Biológicas, Engenharias e Ciências da Computação, Ciências Exatas e da Terra, Humanidades e Ciências da Saúde.

Tabela 1: Número de docentes por unidades - 1995-2002

\begin{tabular}{l|l|l|l|l|l|l|l|l|l|l|l}
\hline Unidade $^{\mathbf{8}}$ & $\mathbf{1 9 9 5}$ & $\mathbf{1 9 9 6}$ & $\mathbf{1 9 9 7}$ & $\mathbf{1 9 9 8}$ & $\mathbf{1 9 9 9}$ & $\mathbf{2 0 0 0}$ & $\mathbf{2 0 0 1}$ & $\mathbf{2 0 0 2}$ & Total & Media & $\mathbf{\%}$ \\
\hline FCM & 419 & 419 & 418 & 411 & 406 & 383 & 375 & 380 & $\mathbf{3 2 1 1}$ & 401,4 & 20,5504 \\
\hline FEF & 52 & 52 & 52 & 48 & 45 & 39 & 35 & 36 & $\mathbf{3 5 9}$ & 44,88 & 2,2976 \\
\hline FOP & 96 & 94 & 94 & 91 & 88 & 88 & 85 & 84 & $\mathbf{7 2 0}$ & 90 & 4,608 \\
\hline IB & 158 & 151 & 146 & 138 & 135 & 128 & 124 & 123 & $\mathbf{1 1 0 3}$ & 137,9 & 7,0592 \\
\hline IMECC & 155 & 162 & 121 & 121 & 112 & 110 & 102 & 106 & $\mathbf{9 8 9}$ & 123,6 & 6,3296 \\
\hline IC & 0 & 43 & 41 & 43 & 43 & 40 & 40 & 40 & $\mathbf{2 9 0}$ & 36,25 & 1,856 \\
\hline IF & 124 & 117 & 109 & 103 & 98 & 94 & 92 & 92 & $\mathbf{8 2 9}$ & 103,6 & 5,3056 \\
\hline IG & 38 & 37 & 43 & 38 & 39 & 42 & 42 & 47 & $\mathbf{3 2 6}$ & 40,75 & 2,0864 \\
\hline IQ & 81 & 82 & 78 & 78 & 76 & 75 & 75 & 74 & $\mathbf{6 1 9}$ & 77,38 & 3,9616 \\
\hline FE & 105 & 112 & 117 & 113 & 108 & 105 & 100 & 106 & $\mathbf{8 6 6}$ & 198,3 & 5,5424 \\
\hline IA & 96 & 115 & 113 & 107 & 97 & 100 & 101 & 106 & $\mathbf{8 3 5}$ & 104,4 & 5,344 \\
\hline IE & 103 & 101 & 99 & 96 & 89 & 81 & 79 & 81 & $\mathbf{7 2 9}$ & 91,13 & 4,6656 \\
\hline IEL & 79 & 80 & 80 & 79 & 73 & 74 & 73 & 74 & $\mathbf{6 1 2}$ & 76,5 & 3,9168 \\
\hline IFCH & 125 & 119 & 117 & 109 & 93 & & 93 & 93 & $\mathbf{7 4 9}$ & 93,63 & 4,7936 \\
\hline FEAGRI & 47 & 45 & 45 & 43 & 41 & 38 & 37 & 38 & $\mathbf{3 3 4}$ & 41,75 & 2,1376 \\
\hline FEC & 78 & 79 & 77 & 77 & 78 & 76 & 73 & 80 & $\mathbf{6 1 8}$ & 77,25 & 3,9552 \\
\hline FEA & 74 & 71 & 70 & 72 & 66 & 66 & 62 & 62 & $\mathbf{5 4 3}$ & 67,88 & 3,4752 \\
\hline FEE & 107 & 110 & 114 & 112 & 110 & 106 & 102 & 103 & $\mathbf{8 6 4}$ & 108 & 5,5296 \\
\hline FEM & 85 & 83 & 81 & 79 & 77 & 76 & 78 & 79 & $\mathbf{6 3 8}$ & 79,75 & 4,0832 \\
\hline FEQ & 42 & 49 & 55 & 49 & 50 & 49 & 48 & 49 & $\mathbf{3 9 1}$ & 48,88 & 2,5024 \\
\hline Total & $\mathbf{2 0 6 4}$ & $\mathbf{2 1 2 1}$ & $\mathbf{2 0 7 0}$ & $\mathbf{2 0 0 7}$ & $\mathbf{1 9 2 4}$ & $\mathbf{1 7 7 0}$ & $\mathbf{1 8 1 6}$ & $\mathbf{1 8 5 3}$ & $\mathbf{1 5 6 2 5}$ & $\mathbf{1 0 2 , 1 6 1 5}$ & $\mathbf{1 0 0}$ \\
\hline
\end{tabular}

FONTE: Unicamp. Anuário de pesquisa 1995-2002.

A quantidade de docentes em cada unidade foi posteriormente comparada com o volume da produção científica, aplicando-se percentagem, de modo que as unidades que contam com menor quantidade de docentes não fossem prejudicadas na somatória mediana. Em relação aos investigadores que participam de grupos de pesquisa, não foi possível identificar o quantitativo, por falta de informação nos anuários de pesquisa da Unicamp.

\footnotetext{
${ }^{8}$ FCM (Faculdade de Ciências Médicas); FEF (Faculdade de Educação Física); FOP (Faculdade de Odontologia de Piracicaba); IB (Instituto de Biologia); IMECC (Instituto de Matemática, Estatística e Ciências da Computação); IC (Instituto de Computação); IF (Instituto de Física); IG (Instituto de Geociências); IQ (Instituto de Química); FE (Faculdade de Educação); IA (Instituto de Artes); IE (Instituto de Economia); IEL (Instituto de Estudos Lingüísticos); IFCH (Instituto de Filosofia e Ciências Humanas); FEAGRI (Faculdade de Engenharia Agrícola); FEC (Faculdade de Engenharia Civil); FEA (Faculdade de Engenharia de Alimentos); FEE (Faculdade de Engenharia Elétrica); FEM (Faculdade de Engenharia Mecânica); FEQ (Faculdade de Engenharia Química).
} 
Para a coleta de dados, primeiramente foi realizado um levantamento dentro dos anuários de pesquisa impressos. A seguir, comparou-se este resultado com as informações contidas na versão em CD-Rom, devido à facilidade de manuseio dos dados, tendo sido priorizada a versão digital. Posteriormente, realizaram-se as buscas no CD-Rom sobre a produtividade científica da Universidade por unidade de ensino.

O passo seguinte foi reunir todas as informações por tipologia documental e por outras atividades, como orientação de teses e dissertações, atividades em grupos de pesquisa e distribuição financeira à investigação (output). Finalmente, compararam-se estes dados com a produção científica (input).

Diante destes resultados, foi realizado um estudo comparativo entre grupos de pesquisas da Unicamp e outras instituições de relevância no âmbito científico do país, servindo para analisar se a instituição atrela seus grupos às suas investigações. Entretanto, esta análise é vista pelo número de grupos existentes (crescentes ou não, no período estudado), pelas produções científicas em geral, indexada no ISI, e pelos títulos de revistas recomendados pelo Qualis/Capes (BRASIL. Coordenação..., 2004). Não foram estudadas as publicações indexadas na base SciELO devido à falta de informação para o período estudado.

Para localizar as publicações usadas pela Unicamp e indexadas na Web of Knowledge (ISI), foi realizada uma averigação de todos estes títulos constantes nos anuários, os quais foram posteriormente comparados com a listagem disponível na ISINet. Para as informações das revistas constantes do Qualis/Capes, seguiu-se o mesmo processo, isto é, por título de revista.

Para que a investigação fosse totalizada, realizou-se também uma coleta quanto ao número de livros publicados, de trabalhos apresentados em eventos e de seminários de iniciação científica.

Com a finalidade de reunir as informações coletadas, foi desenvolvido um sistema (base de dados) em Microsoft Office Access (MICROSOFT, 2003a), sem a preocupação com 
relações, priorizando-se a indexação padronizada dos dados. A configuração gráfica foi confeccionada em Microsoft Office Excel (MICROSOFT, 2003b).

\section{RESULTADOS}

Os principias resultados obtidos a partir dos anuários de pesquisa estão descritos da seguinte forma: grupos de pesquisa; desempenho acadêmico; produção em revistas (nacionais e internacionais); trabalhos apresentados em eventos; e publicação de livros.

As informações coletadas nas memórias de pesquisa permitiram identificar dados importantes sobre a instituição objeto de estudo, como por exemplo que ela consolidou uma quantidade elevada de grupos de investigação.

Ainda segundo os referidos anuários, nos anos de 2001 e 2002, os grupos de pesquisa estavam divididos em $68 \%$ de equipes consolidadas, $27 \%$ de equipes em consolidação e 5\% de equipes em formação. Quanto aos anos anteriores, não havia registro destes percentuais.

Tabela 2: Comparativo dos grupos de pesquisa da Unicamp com as principais universidades brasileiras - 1995-2002.

\begin{tabular}{l|l|l|l|l|l|l|l|l}
\hline \multirow{2}{*}{ Instituição } & $\mathbf{1 9 9 5}$ & $\mathbf{1 9 9 7}$ & $\mathbf{2 0 0 0}$ & $\mathbf{2 0 0 2}$ \\
\cline { 2 - 9 } & Grupos & $\mathbf{9}$ & Grupos & $\mathbf{\%}$ & Grupos & $\mathbf{\%}$ & Grupos & $\mathbf{\%}$ \\
\hline USP & 1.056 & 14,5 & 1.067 & 12,4 & 1.356 & 11,5 & 1.350 & 8,9 \\
\hline UFRJ & 577 & 7,9 & 520 & 6,0 & 679 & 5,8 & 750 & 4,9 \\
\hline UNICAMP & $\mathbf{6 5 9}$ & $\mathbf{9 , 1}$ & $\mathbf{8 1 6}$ & $\mathbf{9 , 5}$ & $\mathbf{5 3 7}$ & $\mathbf{4 , 6}$ & $\mathbf{6 1 4}$ & $\mathbf{4 , 1}$ \\
\hline UNESP & 405 & 5,6 & 369 & 4,3 & 359 & 3,1 & 593 & 3,9 \\
\hline UFRGS & 267 & 3,7 & 355 & 4,1 & 422 & 3,6 & 489 & 3,2 \\
\hline
\end{tabular}

FONTE: Unicamp. Anuário de pesquisa 1995-2002.

O quadro supra, elaborado pela Unicamp, expõe um estudo comparativo com as principias instituições brasileiras de ensino e pesquisa, onde ela apresenta segnificativo desempenho, principalmente em função das atividades dos seus grupos de investigação.

Em 1995, a Unicamp começa a liderar a pesquisa nacional, sediando o Encontro Nacional sobre Pesquisa e Pós-graduação. Esta iniciativa trouxe resultados positivos nos anos seguintes, quando a instituição assina um acordo com a International Business 
Machine (IBM) para a transmissão de imagens do Vaticano, para o desenvolvimento de um estudo sobre a ação biológica da própolis e de padrões para o uso e controle de qualidade de medicamentos (UNICAMP, 2003), concorrendo assim para um avanço significativo na pesquisa brasileira.

Outros relatos da experiência da Unicamp referem-se ao desenvolvimento da fibra ótica e do primeiro satélite de aplicação científica brasileira "Saci I”; a projetos voltados para a região de Campinas; a parceria oficial no projeto Genoma Humano; à conclusão do seqüenciamento dos genes da cana-de-açúcar; à parceria no seqüenciamento do nanochip do ouro, além de outras pesquisas de ponta desenvolvidas no período (BRASIL. Ministério..., 2001).

O quadro científico da Unicamp demonstra sua vocação determinante para a extensão universitária, ao desenvolver projetos multidisciplinares de impacto social. De fato, eles ocupam boa parte da Universidade, estão voltados para problemas reais e para a busca do bem-estar social. Isto se deve, principalmente, ao grande número de pesquisadores ativos que a instituição vem mantendo à frente dos seus projetos: em 1997, havia um total de 1.405 doutores; em 2000, este número subiu para 1.660; e em 2002, chegou a 1.994 doutores ativos na pesquisa, perdendo somente para a Universidade de São Paulo, uma das instituições de maior porte no País.

É evidente o esforço desenvolvido na Unicamp ao longo destes anos, fato que está evidenciado nos indicadores de desempenho acadêmico (Tabela 3), onde a Universidade atingiu números importantes nas publicações indexadas no ISI e apresentou um crescimento expressivo nas atividades de Iniciação Científica. Os seminários internos anuais de Iniciação Científica atraem cerca de 1.500 pessoas e têm se transformado, nos últimos anos, numa verdadeira oportunidade para a captação de novos talentos para os programas de pós-graduação.

Tabela 3: Indicadores de desempenho acadêmico da UNICAMP nos últimos anos. 


\begin{tabular}{l|l|l|l}
\hline Ano & Dissertações e teses & Publicações Indexadas (ISI) & Congresso de Iniciação Científica \\
\hline 1995 & 1.018 & 687 & 354 \\
\hline 1996 & 1.141 & 822 & 350 \\
\hline 1997 & 1.242 & 880 & 350 \\
\hline 1998 & 1.287 & 1.117 & 373 \\
\hline 1999 & 1.363 & 1.229 & 441 \\
\hline 2000 & 1.431 & 1.394 & 517 \\
\hline 2001 & 1.795 & 1.331 & 664 \\
\hline 2002 & 1.864 & 1.636 & 681 \\
\hline
\end{tabular}

FONTE: Unicamp. Anuário de pesquisa 1995-2002.

O aporte de recursos extra-orçamentários tem sido crescente, principalmente daqueles oriundos de empresas privadas, que representam $10 \%$ do total de financiamento externo à pesquisa. Este fato demonstra a forte ligação que a Unicamp vem estabelecendo com o setor produtivo.

A distribuição do financiamento à pesquisa nos últimos oito anos está contabilizada em torno de R\$ 946 milhões, divididos em R\$ 9.490.341,00 da Faep/Unicamp; R\$ 380.450.479,00 da Fapesp; R\$ 199.231.599,00 do CNPq; R\$ 73.067.573,00 da Capes; R\$ 76.518.772,00 de empresas públicas; R\$ 39.302.137,00 da Finep/Pronex; e R\$ 7.647.899,00 de instituições internacionais.

Por conta destes recursos captados, a instituição logra gerar indicadores significativos de output, o que lhe confere uma forte visibilidade no sistema ISI (Social Science Citation Index e Science Citation Index) e nas revistas categorizadas em tipo $\mathrm{A}^{9}$ no programa Qualis/Capes, como se pode visualizar no gráfico 1.

\footnotetext{
${ }^{9}$ Corresponde às revistas especializadas de grande circulação internacional e/ou nacional.
} 


\section{Gráfico 1: Produção em revistas científicas nacionais e internacionais (tipo A).}

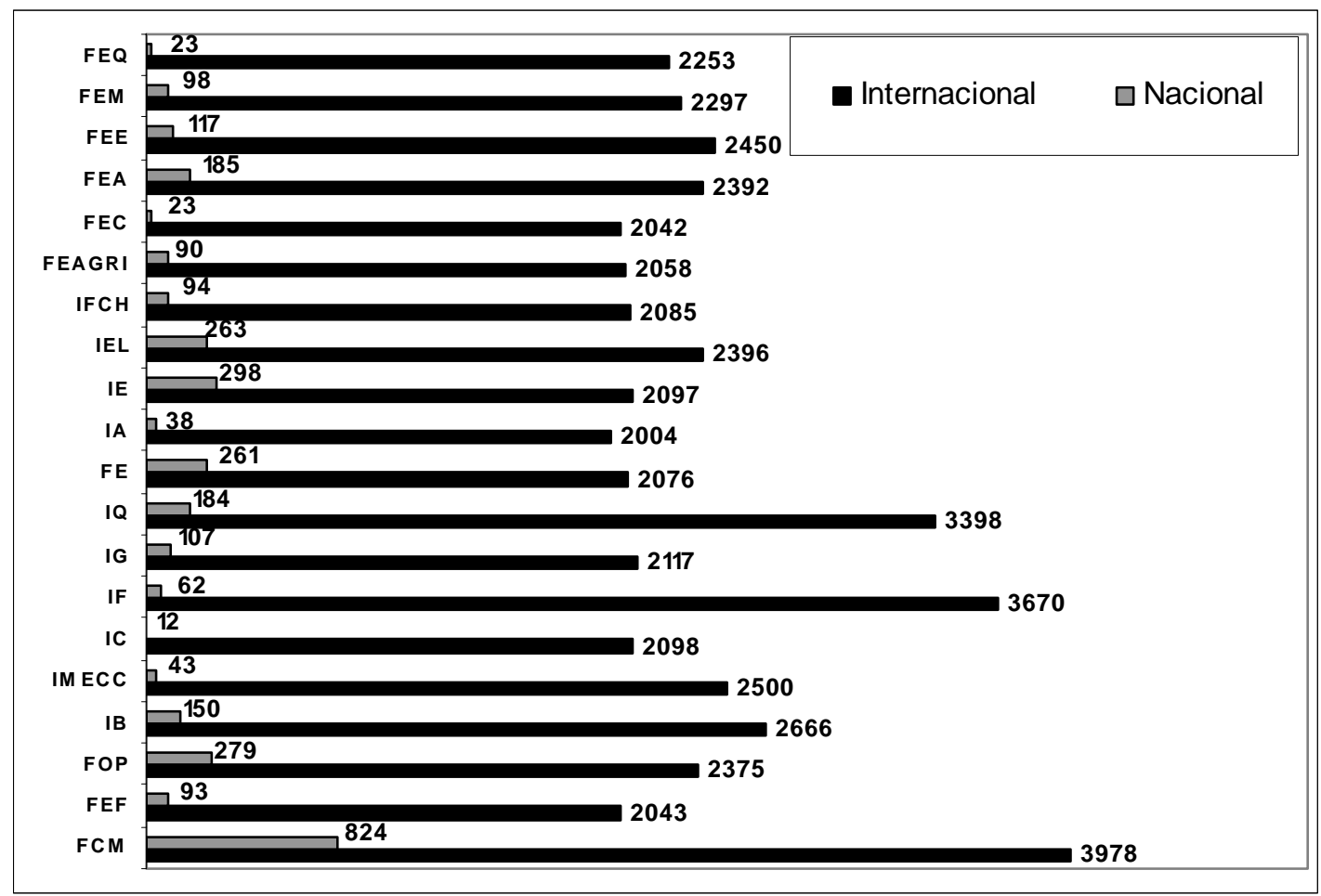

FONTE: Unicamp. Anuário de pesquisa 1995-2002.

Indiscutivelmente, a Faculdade de Ciências Médica, o Instituto de Física e o Instituto de Química são as unidades que mais produziram no período. Também foram as que mais receberam investimentos, além de contarem com um número elevado de docentes em relação às outras (FCM, média de 400 docentes/ano; IF, média de 103 docentes/ano; IQ, média de 104 docentes/ano). Paralelamente, estas três unidades apresentam uma quantidade elevada de projetos financiados por agências de fomento (FCM, 126 projetos/ano; IF, 73 projetos/ano; IQ, 77 projetos/ano). Outras unidades apresentaram índices muito abaixo nesta relação, com exceção do Instituto de Biologia (IB), que conta com um número maior de docentes e uma quantidade mais elevada de projetos que IF e IQ e, mesmo assim, não conseguiu publicar muitos artigos em revistas internacionalmente indexadas.

Uma curiosidade em relação à produção científica e ao desenvolvimento de projeto é que todas as unidades tiveram um número muito baixo em projetos consolidados no ano de 1995, comparando-se com os demais anos, quando a produção foi muito elevada. 
Em todo o período estudado, os anos de 1998, 1999 e 2002 apresentaram os maiores índices de produção, com aproximadamente 1.450 trabalhos publicados em revistas internacionais e com cerca de 420 , em revistas nacionais.

Com exceção da FCM, IF e IQ, as demais unidades tiveram médias de docentes e de projetos muito semelhantes e um índice de pelo menos 2.000 trabalhos publicados em revistas internacionais. Este fator demonstra a constante preocupação da Unicamp em divulgar seus trabalhos fora do Brasil, principalmente porque muitos deles constituem pesquisa de ponta em âmbito mundial, como a análise de genomas, Nanotecnologia, Inteligência Artificial e Ontologia, áreas em que a Unicamp desfruta de respeitabilidade internacional.

Além das publicações classificadas como tipo A, também foram estudadas as publicações de categoria $B$, isto é, revistas com menor expressão que as primeiras. No entanto, esta classificação não está associada, necessariamente, à qualidade científica e formal das revistas e, sim, à sua temática.

\section{Gráfico 2: Produção de categoria B.}

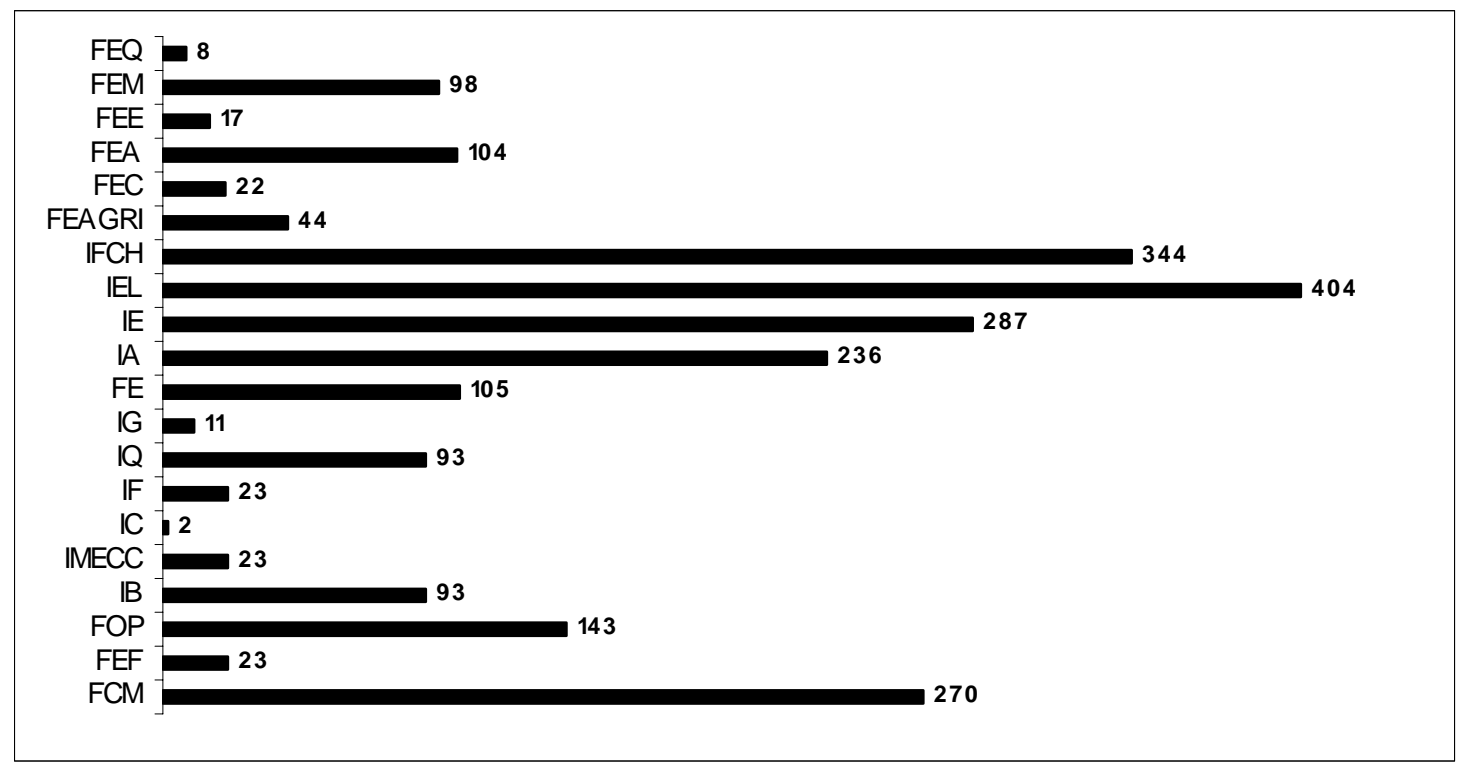

FONTE: Unicamp. Anuário de pesquisa 1995-2002.

Assim, quanto às revistas de tipo $B$, os resultados deste estudo indicam as unidades que mais publicaram foram o Instituto de Estudos Lingüísticos, o Instituto de Filosofia e o 
Instituto de Filosofia e Ciências Humanas, o Instituto de Economia, a Faculdade de Ciências Médica e o Instituto de Artes.

O pico de maior incidência de produção neste tipo de publicação aconteceu em 1998, com 446 trabalhos publicados; em 2000 e 2001, com 463 trabalhos publicados cada ano; e 2002, com 570 trabalhos publicados em toda a Universidade. Para os demais anos, a produção foi um pouco mais discreta, com 24 trabalhos em 1995, 62 em 1996, 72 em 1997 e 249 em 1999.

Outra análise realizada se refere aos trabalhos apresentados em eventos (congressos, colóquios, encontros e simpósios). De fato, muitos destes trabalhos trazem consigo a mesma complexidade dos artigos científicos quanto à sua elaboração. No entanto, para efeito de avaliação por parte dos órgãos que controlam o ensino e a pesquisa nacional, eles apresentam baixa pontuação. Mesmo assim, considerou-se interessante avaliar estas publicações, como se pode visualizar no gráfico 3, a seguir.

\section{Gráfico 3: Trabalhos publicados em eventos científicos nacionais e internacionais.}

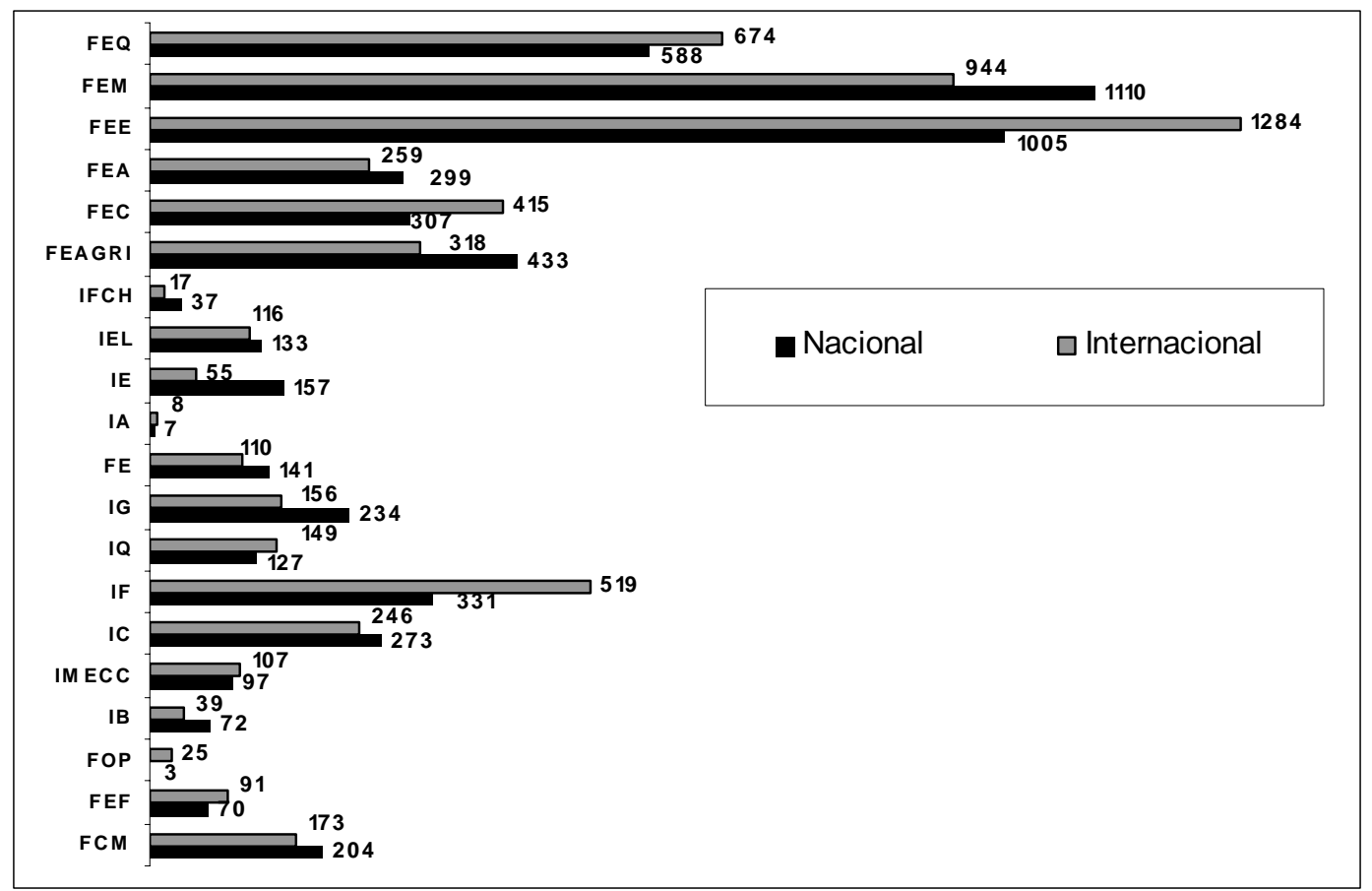

FONTE: Unicamp. Anuário de pesquisa 1995-2002. 
Assim como se observa no gráfico anterior, as unidades que mais divulgaram neste tipo de publicação foram a Faculdade de Engenharia Elétrica e de Computação ${ }^{10}$, seguida pela Faculdade de Engenharia Mecânica ${ }^{11}$ e Faculdade de Engenharia Química ${ }^{12}$. As demais unidades conseguiram número menos significativo, com exceção do Instituto de Física e da Faculdade de Engenharia Civil que, conseguiram índices bem próximos, embora com muitas variações de um ano para outro, não conseguindo estabelecer uma média no período estudado.

Outro ponto enfocado na presente pesquisa foi a análise dos livros editados e publicados no período estudado, que fazem parte da literatura branca da Unicamp.

Tabela 4: Livros publicados pelas unidades por ano.

\begin{tabular}{l|l|l|l|l|l|l|l|l|l}
\hline Livros & $\mathbf{1 9 9 5}$ & $\mathbf{1 9 9 6}$ & $\mathbf{1 9 9 7}$ & $\mathbf{1 9 9 8}$ & $\mathbf{1 9 9 9}$ & $\mathbf{2 0 0 0}$ & $\mathbf{2 0 0 1}$ & $\mathbf{2 0 0 2}$ & Total \\
\hline FCM & 7 & 27 & 11 & 21 & 19 & 7 & 7 & 8 & $\mathbf{1 0 7}$ \\
\hline FEF & 9 & 16 & 6 & 9 & 5 & 10 & 10 & 3 & $\mathbf{6 8}$ \\
\hline FOP & 0 & 0 & 0 & 2 & 0 & 2 & 2 & 2 & $\mathbf{8}$ \\
\hline IB & 2 & 14 & 2 & 0 & 3 & 2 & 2 & 1 & $\mathbf{2 6}$ \\
\hline IMECC & 9 & 2 & 3 & 3 & 4 & 2 & 2 & 3 & $\mathbf{2 8}$ \\
\hline IC & 0 & 2 & 2 & 4 & 1 & 1 & 1 & 2 & $\mathbf{1 3}$ \\
\hline IF & 4 & 2 & 5 & 2 & 2 & 1 & 1 & 1 & $\mathbf{1 8}$ \\
\hline IG & 4 & 8 & 8 & 4 & 1 & 7 & 7 & 7 & $\mathbf{4 6}$ \\
\hline IQ & 1 & 1 & 0 & 2 & 1 & 3 & 3 & 1 & $\mathbf{1 2}$ \\
\hline FE & 15 & 24 & 22 & 33 & 24 & 41 & 41 & 25 & $\mathbf{2 2 5}$ \\
\hline IA & 5 & 4 & 0 & 1 & 7 & 5 & 5 & 5 & $\mathbf{3 2}$ \\
\hline IE & 15 & 13 & 14 & 16 & 4 & 14 & 14 & 12 & $\mathbf{1 0 2}$ \\
\hline IEL & 14 & 25 & 28 & 22 & 17 & 22 & 22 & 39 & $\mathbf{1 8 9}$ \\
\hline IFCH & 28 & 30 & 8 & 1 & 3 & 22 & 22 & 33 & $\mathbf{1 4 7}$ \\
\hline FEAGRI & 2 & 5 & 2 & 2 & 4 & 2 & 2 & 1 & $\mathbf{2 0}$ \\
\hline FEC & 1 & 5 & 0 & 0 & 3 & 0 & 0 & 1 & $\mathbf{1 0}$ \\
\hline FEA & 5 & 13 & 3 & 3 & 17 & 2 & 2 & 0 & $\mathbf{4 5}$ \\
\hline FEE & 2 & 1 & 2 & 4 & 5 & 1 & 1 & 0 & $\mathbf{1 6}$ \\
\hline FEM & 1 & 0 & 3 & 3 & 2 & 4 & 4 & 0 & $\mathbf{1 7}$ \\
\hline FEQ & 0 & 0 & 0 & 0 & 0 & 1 & 1 & 0 & $\mathbf{2}$ \\
\hline Total & $\mathbf{1 2 4}$ & $\mathbf{1 9 2}$ & $\mathbf{1 1 9}$ & $\mathbf{1 3 2}$ & $\mathbf{1 2 2}$ & $\mathbf{1 4 9}$ & $\mathbf{1 4 9}$ & $\mathbf{1 4 4}$ & $\mathbf{1 1 3 1}$ \\
\hline
\end{tabular}

FONTE: Unicamp. Anuário de pesquisa 1995-2002.

Tem-se que as unidades que mais publicaram livros foram FE (225), seguida pelo IEL (189), IFCH (147), FCM (107) e IE (102), somando 68,06\% do total de livros

\footnotetext{
${ }^{10}(1995$ - 294 / 1996 - 340 / 1997- 246 / 1998 - 262 / 1999 - 282 / 2000 - 269 / 2001 - 269 / 2002 - 327)

${ }^{11}(1995-203$ / 1996 - 304 / 1997- 320 / 1998 - 262 / $1999-305$ / $2000-196$ / 2001 - 196 / 2002 - 268)

12 (1995 - 116 / 1996 - 201 / 1997- 62 / 1998 - 145 / 1999 - 105 / 2000 - 191 / 2001 - 191 / 2002 - 161)
} 
publicados no período. O volume maior está concentrado na área das Humanidades, cujas sub-áreas trabalham com extensa temporalidade e grande carga teórica.

Finalizando este estudo, é fundamental visualizar o quadro geral de produtividade da Unicamp, comparada com a média de professores por área/ano, como pode-se observar na tabela 5.

Tabela 5: Quadro geral da produção científica e da porcentagem de autores.

\begin{tabular}{l|l|l|l|l|l|l|l|l|l|l}
\hline Unidade & $\begin{array}{l}\text { Media } \\
\text { Autores }\end{array}$ & $\mathbf{9}$ & $\begin{array}{l}\text { E. } \\
\text { N. }\end{array}$ & $\mathbf{E . ~ I . ~}$ & $\begin{array}{l}\text { Revistas } \\
\mathbf{A}(\mathbf{N})\end{array}$ & $\begin{array}{l}\text { Revistas } \\
\mathbf{A}(\mathbf{I})\end{array}$ & $\begin{array}{l}\text { Revistas } \\
\mathbf{B}\end{array}$ & Livros & Total & $\begin{array}{l}\text { P.C. / } \\
\text { Autor/Ano }\end{array}$ \\
\hline FCM & 401,4 & 20,5504 & 204 & 173 & 824 & 3978 & 270 & 107 & $\mathbf{5 5 5 6}$ & $\mathbf{1 , 4}$ \\
\hline FEF & 44,88 & 2,2976 & 70 & 91 & 93 & 2043 & 23 & 68 & $\mathbf{2 3 8 8}$ & $\mathbf{6 , 3}$ \\
\hline FOP & 90 & 4,608 & 3 & 25 & 279 & 2375 & 143 & 8 & $\mathbf{2 8 3 3}$ & $\mathbf{3 , 6}$ \\
\hline IB & 137,9 & 7,0592 & 72 & 39 & 150 & 2666 & 93 & 26 & $\mathbf{3 0 4 6}$ & $\mathbf{2 , 5}$ \\
\hline IMECC & 123,6 & 6,3296 & 97 & 107 & 43 & 2500 & 23 & 28 & $\mathbf{2 7 9 8}$ & $\mathbf{2 , 5}$ \\
\hline IC & 36,25 & 1,856 & 273 & 246 & 12 & 2098 & 2 & 13 & $\mathbf{2 6 4 4}$ & $\mathbf{8 , 8}$ \\
\hline IF & 103,6 & 5,3056 & 331 & 519 & 62 & 3670 & 23 & 18 & $\mathbf{4 6 2 3}$ & $\mathbf{5 , 2}$ \\
\hline IG & 40,75 & 2,0864 & 234 & 156 & 107 & 2117 & 11 & 46 & $\mathbf{2 6 7 1}$ & $\mathbf{7 , 9}$ \\
\hline IQ & 77,38 & 3,9616 & 127 & 149 & 184 & 3398 & 93 & 12 & $\mathbf{3 9 6 3}$ & $\mathbf{6 , 1}$ \\
\hline FE & 198,3 & 5,5424 & 141 & 110 & 261 & 2075 & 105 & 225 & $\mathbf{2 9 7 1}$ & $\mathbf{1 , 5}$ \\
\hline IA & 104,4 & 5,344 & 7 & 8 & 38 & 2004 & 236 & 32 & $\mathbf{2 3 2 5}$ & $\mathbf{2 , 5}$ \\
\hline IE & 91,13 & 4,6656 & 157 & 55 & 298 & 2097 & 287 & 102 & $\mathbf{2 9 9 6}$ & $\mathbf{3 , 8}$ \\
\hline IEL & 76,5 & 3,9168 & 133 & 116 & 263 & 2396 & 404 & 189 & $\mathbf{3 5 0 1}$ & $\mathbf{5 , 4}$ \\
\hline IFCH & 93,63 & 4,7936 & 37 & 17 & 94 & 2085 & 344 & 147 & $\mathbf{2 7 2 4}$ & $\mathbf{3 , 3}$ \\
\hline FEAGRI & 41,75 & 2,1376 & 433 & 318 & 90 & 2058 & 44 & 20 & $\mathbf{2 9 6 3}$ & $\mathbf{8 , 5}$ \\
\hline FEC & 77,25 & 3,9552 & 307 & 415 & 23 & 2042 & 22 & 10 & $\mathbf{2 8 1 9}$ & $\mathbf{4 , 2}$ \\
\hline FEA & 67,88 & 3,4752 & 299 & 259 & 185 & 2392 & 104 & 45 & $\mathbf{3 2 8 4}$ & $\mathbf{5 , 7}$ \\
\hline FEE & 108 & 5,5296 & 1005 & 1284 & 117 & 2459 & 17 & 16 & $\mathbf{4 8 9 8}$ & $\mathbf{5 , 4}$ \\
\hline FEM & 79,75 & 4,0832 & 1110 & 944 & 98 & 2297 & 98 & 17 & $\mathbf{4 5 6 4}$ & $\mathbf{6 , 8}$ \\
\hline FEQ & 48,88 & 2,5024 & 588 & 674 & 23 & 2253 & 8 & 2 & $\mathbf{3 5 4 8}$ & $\mathbf{8 , 7}$ \\
\hline Total & $\mathbf{1 9 5 3 , 1 2 5}$ & $\mathbf{1 0 0}$ & $\mathbf{5 6 2 8}$ & $\mathbf{5 7 0 5}$ & $\mathbf{3 2 4 4}$ & $\mathbf{4 9 0 0 3}$ & $\mathbf{2 3 5 0}$ & $\mathbf{1 1 3 1}$ & $\mathbf{6 7 0 6 1}$ & $\mathbf{1 0 0}$ \\
\hline
\end{tabular}

FONTE: Unicamp. Anuário de pesquisa 1995-2002.

Legenda: E.N. (Trabalhos publicados em eventos nacionais); E.I. (Trabalhos publicados em eventos internacionais); N (Nacional); I (Internacional); P.C. (Produção Científica).

Em toda a análise, identificou-se a produtividade científica, desconsiderando a comparação entre a soma de todos os trabalhos publicados, dividida pela média de professores por unidade.

Neste tipo de análise, as áreas que apresentaram maior produtividade em números absolutos não conseguiram uma equivalência reveladora de sua situação. Neste caso, encontra-se a FCM, que teve uma media de 1,4 trabalhos firmados por professor. O que se quer explicar é que o somatório dos dados é fundamental, porém, só quando se 
calcula a média de publicações por professor é que se consegue identificar a real situação da área.

A ordem das áreas que apresentaram um forte índice neste aspecto - publicação por professor - é: IC com 8,8 trabalhos/docente; FEQ com 8,7 trabalhos/docente; FEAGRI com 8,5 trabalhos/docente; IG com 7,9 trabalhos/docente; FEM com 6,8 trabalhos/docente; FEF com 6,3 trabalhos/docente; IQ com 6,1 trabalhos/docente; FEA com 5,7 trabalhos/docente; FEE com 5,4 trabalhos/docente; IEL com 5,4 trabalhos/docente; IF com 5,2 trabalhos/docente; FEC com 4,2 trabalhos/docente; IE com 3,8 trabalhos/docente; FOP com 3,6 trabalhos/docente; IFCH com 3,3 trabalhos/docente; IB com 2,5 trabalhos/docente; IMECC com 2,5 trabalhos/docente; IA com 2,5 trabalhos/docente; FE com 1,5 trabalhos/docente; e FCM com 1,4 trabalhos/docente.

\section{CONCLUSÃO}

O que se tentou evidenciar nesse artigo foi à representatividade científica da Universidade Estadual de Campinas, ao serem identificados os seguintes aspectos:

- A Unicamp detém um número elevado de grupos de pesquisa em comparação às principais universidades brasileiras, contando também com elevado índice de pesquisadores (docentes e investigadores) envolvidos na atividade;

- A instituição manteve um índice significativo em teses defendidas e uma forte visibilidade no ISI, conforme se observa na tabela 3;

- A publicação de artigos em revistas do tipo A teve principal destaque nas unidades FCM, IF, IQ e IB, porém o IMECC, FEE, FEA, FOP, FE e o IE apresentaram um índice significativo, isto é, acima de 2.000 trabalhos em revistas internacionais e 290 trabalhos em revistas nacionais;

- Os artigos publicados em revistas do tipo B tiveram seu principal destaque nas unidades IEL, IFCH, IE, FCM e IA, com uma média superior a 220 trabalhos firmados;

- Os trabalhos publicados em eventos foram dominados pelas unidades de Engenharia, onde se pode identificar que das seis mais produtoras cinco 
pertencem a esta área (FEE, FEM, FEQ, IF, FEAGRI e FEC), com uma média acima de 720 trabalhos;

- Quanto à análise da publicação de livros, pode-se destacar que os maiores produtores neste segmento foram FE, IEL, IFCH, FCM e IE, com um índice de pelos menos 100 livros publicados no período estudado.

No quadro geral da investigação realizada, destaca-se ainda que muitas unidades apresentaram elevado índice de publicações, devido ao número de docentes e investigadores, porém em uma análise percentual estas áreas não conseguiram o mesmo destaque. A unidade FCM, sempre entre as mais positivas na Unicamp neste tipo de análise, não apresentou elevado índice publicação com relação ao total de docentes e investigadores. Porém, algumas unidades que quase não se destacaram em toda a análise tiveram um índice muito elevado, como é o caso do IC.

Ainda se pode afirmar que as unidades mais produtivas, isto é, número de publicações divididas por número de docentes e investigadores, são IC, seguido por FEQ, FEAGRI, IG, FEM, FEF, IQ, FEA, FEE, IEL, IF, FEC, IE, FOP, IFCH, IB, IMECC, IA, FE e FCM.

\section{REFERÊNCIAS}

BRASIL. Coordenação de Aperfeiçoamento de Pessoal de Nível Superior. Avaliação: Qualis das áreas. Brasília: Capes, 2004. Disponível em: <http://qualis.capes.gov.br/>. Acesso em: 05 mar. 2005.

BRASIL. Ministério de Desenvolvimento, Indústria e Comércio. Cronologia do desenvolvimento científico e tecnológico brasileiro (1950-2000). Brasília: Secretaria de Tecnologia Industrial, 2001.

GARFIELD, E. Citation indexes for science: A new dimension in documentation through association of ideas. Science, Washington, v. 122, n. 3159, p. 108-111, July 1955.

LOTKA, A. J. The frequency distribution of scientific productivity. Journal of the Washington Academy of Sciences, Washington, v. 16, n. 12, 1926, p. 317-323.

LOUZADA, R. C. R. Sobre o crescimento da produtividade científica brasileira: anotações. Revista de Espaço Acadêmico, São Paulo, a. 2, n. 18, novembro, 2002.

MICROSOFT. Access Office Professional: Windows 2000/XP. Madrid: Microsoft, 2003a. (Versão em CD-Rom). 
MICROSOFT. Excel Office Professional: Windows 2000/XP. Madrid: Microsoft, 2003b. (Versão em CD-Rom).

NATURE destaca produção científica do Brasil. Jornal da Unicamp, Campinas, v. 17, n. 192, 30 set -6 out. 2002.

OHIRA, M. L. B. Produção técnico-científica e artística da universidade do Estado de Santa Catarina (1991-1995). In: WITTER, G. P. Produção científica. Campinas: Átomo, 1997, p. 87- 113.

PEREIRA, Dl. No topo do ranking per capita, produção científica da Unicamp pode crescer mais. Jornal da Unicamp, Campinas, n. 296, 8-14 ago. 2005.

POBLACIÓN, D. A.; NORONHA, D. P.; CURRÁS, E. Literatura cinzenta versus literatura branca: transição dos autores das comunicações dos eventos para produtores de artigos. Ciência da Informação, Brasília, v. 25, n. 2, 1995, p. 3-13.

PRICE, D. J. S. Little science, big science. New York: Columbia University, 1963.

SCHWARTZMAN, S. Repensando o desenvolvimento: educação, ciência, tecnologia. In: SEMINÁRIO DESENVOLVIMENTO E A POLÍTCA DE DESENVOLVIMENTO,1993, São Paulo. Disponível em URL: <http://www.schwartzman.org.br/simon/goethe.htm>. Acesso em: 20 de outrubro de 2003.

UNICAMP. Anuário de pesquisa 1995-2002. Campinas, 2003. 8 CD-ROMs. 


\section{Adilson Luiz Pinto}

Doutorando em Documentação pela Universidad Carlos III de Madrid; Mestre em Ciência de Informação pela Pontifícia Universidade Católica de Campinas.

100059034@alumnos.uc3m.es - didis98@hotmail.com

\section{Beatriz-Ainhize Rodriguez Barquín}

Doutoranda em Documentação pela Universidad Carlos III de Madrid; Mestre em Documentação Audiovisual pela Universidad Carlos III de Madrid. beatriz.rodriguez@uc3m.es

Recebido em: 08/10/2005

Aceito para publicação em: jun.2006 